\title{
An innovative Telemedicine Initiative implemented with limited resources during COVID-19 pandemic in Sri Lanka
}

\author{
Indika Karunathilake ${ }^{1}$ \\ ${ }^{1}$ Affiliation not available
}

January 11, 2022

\section{Introduction}

Sri Lanka is a lower-middle income country in the South Asian region. Sri Lanka's COVID-19 response is characterised by a strong focus on a preventive approach with rational utilization of available resources. For most of the course of the pandemic, all patients who tested positive were managed at healthcare institutions. Asymptomatic or mildly symptomatic patients were admitted to Intermediate Care Centres for monitoring and symptomatic management and discharged in 14 days. Those with marked symptoms, uncontrolled comorbidities and other complications were admitted to hospitals for closer monitoring and specialized management ${ }^{1,2}$.

Even though this approach proved efficient during the initial phase of the pandemic in 2020, it was sustainable due to the rapid rise in case numbers since July 2021. During the peak of the surge, the number of daily reported test positive patients exceeded 5000 (220 cases per million population and ranked $50^{\text {th }}$ in the world-wide affected country list) and the actual number of estimated new patients per day was around 6000 to $9000^{2,3}$. Even an increase of the number of beds allocated for COVID-19 patients to 34000 was not adequate. Increase in number of deaths was unprecedented. The number of healthcare workers available for the functioning of the system too declined with a significant proportion acquiring the infection and the remaining workforce on the verge of physical and mental exhaustion. The healthcare system of the country was reaching a tipping point ${ }^{4,5}$. The priority shifted towards triaging and identifying those who need immediate medical intervention at the earliest stage of the disease to reduce severe disease and death. This was necessary to prevent overburdening the already fraught hospitals as well as staff and to conserve bed space for severely affected or vulnerable patients who needed specialized care in hospitals ${ }^{3}$.

\section{Intervention}

A virtual triaging system for the identification and evacuation of those who needed hospitalization and to facilitate home management for mild and asymptomatic patients was proposed. This article presents an integrated patient management system established via short message services (SMSs) and telephone helplines, with the involvement of multiple stakeholders. Four key stakeholders, the National Operation Centre for Prevention of COVID-19 Outbreak (NOCPCO), Sri Lanka Medical Association (SLMA), the Ministry of Health and National Ambulance Service were brought together with the cooperation and support of national telecommunication service providers.

The approach was to integrate and expand on existing services. The 247 Doc Call Service under the SLMA was already in operation to provide advice for COVID-19 patients since 2020. The ambulance service, "Suwaseriya", was operating under the toll-free telephone code of 1990. In addition to the above services, a SMS gateway with the toll-free telephone code 1904 was established in NOCPCO.

The integrated system was established to coordinate the abovementioned separate operations to achieve the following objectives: 
(a) Reduce the number of non-urgent admissions to the hospitals,

(b) Provide correct information to patients

(c) Identify and evacuate patients who need urgent care to hospitals.

As the number implies 247 is a twenty-four hour seven days of the week service, made available in all three national languages, Sinhala, Tamil and English used in Sri Lanka. All medical professionals rendered their services on a volunteer basis.

The 1904 SMS service was for COVID patients to contact the National Operations Centre for help. The SMS service was available even in a basic mobile phone and could be operated by a patient without much skill. The general public was informed through media to submit their basic details via an SMS. The symptom profiles were categorized as A, B and C for urgent action (Shortness of breath was designated as A, other symptoms by $\mathrm{B}$ and asymptomatic test positivity by $\mathrm{C}$ ). The patients start the message typing the letters $\mathrm{A}$, $\mathrm{B}$ or $\mathrm{C}$ based on their perceived symptoms. Information based on SMS is immediately directed to a medical team stationed at the operations room of the NOCPCO, where the patient is evaluated over the phone and decisions were made on the necessity for urgent evacuation to a hospital. The details of those who need urgent medical care were notified to the ambulance system and a focal point in the Ministry of Health for allocation of a hospital bed for urgent evacuation.

Category B were triaged and those who required medical attention were transferred to an appropriate facility through a health-military coordinated evacuation mechanism. Category $\mathrm{C}$ patients were given necessary medical advice to remain at home with the provision of information for self-care. Pulse oximeters for self-monitoring at home were distributed free of charge to the needy patients. This integrated system is summarized in Figure 1.

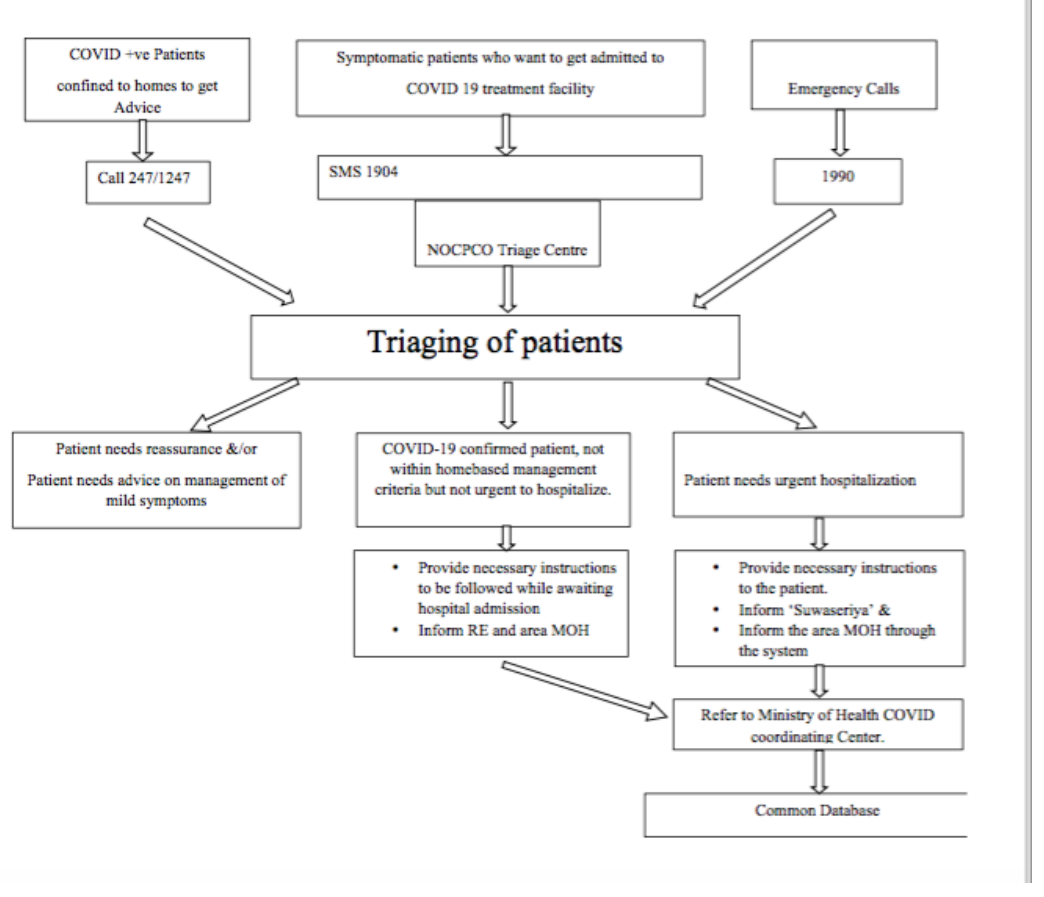

Figure 01 - General pathway of the integrated patient helpline system

\section{Impact of the integrated information system on healthcare services}

The integrated helpline system described above had a major impact on the management of the recent surge 
of COVID-19 pandemic in the country. This portal ensures that those who required hospitalization were given priority for evacuation, and those asymptomatic or mildly symptomatic were given necessary advice.

So far, up to 14th November 2021, Doc call 247 has attended to over 60,000 patients and over 30,000 SMSs via 1904. Out of the 60,000 calls received on Doc call 247, only around 1,300 patients needed urgent care and hospitalization. The waiting time based on system information was $1.5-2$ minutes for the medical team to connect with a patient calling or sending a SMS. If the waiting time exceeded two minutes, the members of the Coordinating Committee would inform additional volunteers to join the system. During the peak of the surge, there was a $70 \%$ response rate for Doc call 247 service. A list of missed calls, if any, is taken every few hours and then transferred to 1904 system where a separate set of doctors would call the patients and assess them. The number of 247 calls responded to and emergency evacuations carried out via 1990 ambulance system over time is provided in figure 2 a.

Through these services it was possible to operate an effective virtual triaging system and limit admissions only to those who needed urgent care. Within two weeks of commencement of the integrated service, an increase the number of available beds in both the government and private sector was observed. Figure 02b illustrates the reduction in hospital admissions, oxygen dependency, comorbidities and death count, following the introduction of the integrated helpline system. It also reduced the unnecessary exposure of healthcare workers to infected individuals, which in turn ensured that medical staff had lesser burdens while carrying out their duties.
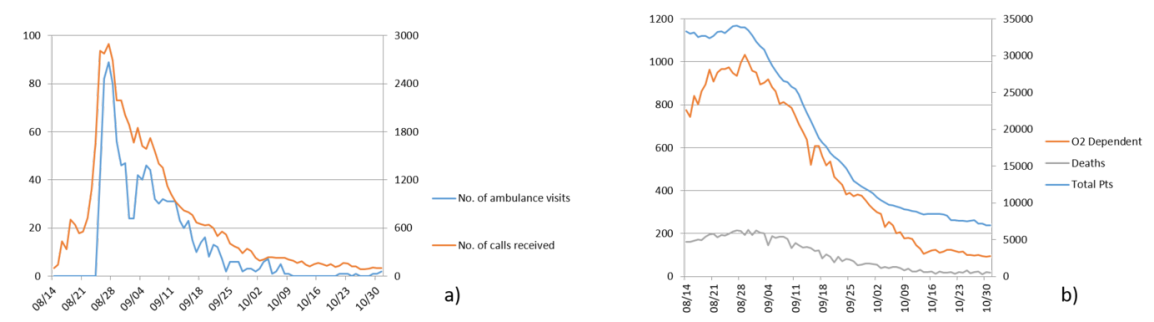

Figure 02 - a) The number of 247 calls responded and emergency evacuations (14.08.2021- 31.10.2021) b) Reduction of Hospital admissions/O2 dependency and Deaths (14.08.2021- 31.10.2021)

\section{Challenges and Sustainability}

Although many countries have attempted diverse methods to overcome the challenges imposed by the pandemic, there is lack of evidence on establishment of patient helpline systems in a low-resource setting. Implementation of patient helpline systems in a low-resource context involves unique challenges, including financial, logistical, administrative and technological ${ }^{4-8}$.

Orchestrating the massive communication process was a challenge due to unpreparedness of doctors, chaotic situation created by the increasing number of cases and deaths on a daily basis, uncertainties of the disease condition itself and fear psychosis created by the media. Patients were distressed not only by their own illness but also by the potential plight of their family members. The entire scenario created the highest possible challenge in communication ${ }^{8}$.

Training a diverse group of volunteers to work in a complex system was a major challenge. Doctors were trained via an online training programme and motivated to share knowledge among them through a dedicated social media platform. This was a display of connectivism; the learning paradigm of digital era ${ }^{10}$.

Sustainable coordination was a major concern. Doc call 247 service consists entirely of volunteers ranging from specialists to medical students. They can tune into the system as per their convenience by 'opting in' or 'opting out. There is a roster system in place as well to ensure that a particular number of doctors are available at a particular time. Number of expatriate medical professionals also rendered their services as volunteers to sustain the helpline transforming this operation to a global telemedicine project ${ }^{5,6}$. Operating 
a SMS gateway for patient prioritization needs a dedicated medical team stationed at an operational room to respond to the SMSs received.

Therefore, this integrated system was a unique example of medical voluntarism. People displayed global empathy; compassion and collaboration for the benefit of people whom they have not met or were not responsible for.

There were some concerns regarding confidentiality of medical information sharing on the web and data security. The issues of liability, jurisdiction and malpractice where medical advice without face-to-face consultation or clinical examination took place, were raised. Some healthcare professionals were reluctant to adapt to new ways of working, resistant to a change in mind-set about the use of technology to deliver health services and lacked willingness to work in multi-disciplinary teams or to ask for a second opinion.

\section{Conclusion}

The innovative Telemedicine Initiative from Sri Lanka utilized the volunteerism in a resource-constrained scenario during COVID-19 pandemic and harnessed the support from all stakeholders including sectors previously underutilized in the provision of healthcare, including information and communication systems. This project has been able to integrate its healthcare system and volunteer professionals by technology to face the challenge. This has made contributions to reduce the burden on treatment centres and healthcare professionals and ensure equity in the distribution of its resources. This innovative approach to healthcare provision is a model to follow in an unprecedented crisis situation in anywhere in the world.

The pandemic may be considered as a wake-up call to utilize information and communication technologies effectively, going forward to provide healthcare and reduce the burden on healthcare institutions and professionals ${ }^{4-9}$.

\section{Acknowledgements}

The head and staff of National Operational Center for Prevention of COVID 19 Outbreak (NOCPCO), Sri Lanka Medical Association, Mobitel National Telecommunication system, Ministry of Health officials, all doctors, Experts and Medical students who volunteered to provide their service, team of Suwaseriya ambulance service for their contribution towards the success of this initiative.

\section{References}

1. Karunathilake I, Mudiyanse R, Witharana C, Peiris M. The rational approach for controlling the spread of COVID-19 in Sri Lanka. Ceylon Medical Journal . 2020;65(3)

2. Karunathilake, I.; Amarasiri, M.; Hamdani, A. Application Of Statistical Modeling To Interpret A Health System Crisis In Sri Lanka Due To COVID- 19. Preprints 2021, 2021120455 (doi: 10.20944/preprints202112.0455.v1).

3. Ministry of Health. COVID-19 Situational Analysis Dashboard. 2021http://wwwhealthgovlk/moh_final/english/public/elfinder/files/library/Statisticshtml.

4. Kristal R, Rowell M, Kress M, et al. A Phone Call Away: New York's Hotline And Public Health In The Rapidly Changing COVID-19 Pandemic: A descriptive commentary about New York City Health+ Hospitals clinician-staffed COVID-19 hotline.Health Affairs . 2020;39(8):1431-1436.

5. Jensen T, Holgersen MG, Jespersen MS, et al. Strategies to handle increased demand in the COVID-19 crisis: a coronavirus EMS support track and a web-based Self-Triage system.Prehospital Emergency Care . 2020;25(1):28-38.

6. Wang J, Wei H, Zhou L. Hotline services in China during COVID-19 pandemic. Journal of Affective Disorders . 2020;275:125. 
7. Choi YS, Ko JY. Operating a national hotline in Korea during the COVID-19 pandemic. Osong Public Health and Research Perspectives . 2020;11(6):380

8. Hegde SKB, Sharma M, Alam I, Bambrah HS, Deshmukh A, Varma D. Leveraging Health Helplines to Combat COVID-19 Pandemic: A Descriptive Study of Helpline Utilization for COVID-19 in 8 States of India. Asia Pacific Journal of Public Health . 2021:10105395211001173.

9. Hardavella G, Aamli-Gaagnat A, Frille A, Saad N, Niculescu A, Powell P. Top tips to deal with challenging situations: doctor-patient interactions. Breathe . 2017;13(2):129-135

10. Goldie JGS. Connectivism: A knowledge learning theory for the digital age? Medical teacher. 2016;38(10):1064-1069. 